\title{
Influence of Chemically Reacting Ferromagnetic Carreau Nanofluid over a Stretched Sheet with Magnetic Dipole and Viscous Dissipation
}

\author{
R. M. Akram Muntazir, ${ }^{1}$ M. Mushtaq, ${ }^{1}$ S. Shahzadi, ${ }^{2}$ and K. Jabeen ${ }^{1}{ }^{1}$ \\ ${ }^{1}$ Department of Math, UET, Lahore, Pakistan \\ ${ }^{2}$ Department of Physics, UET, Lahore, Pakistan \\ Correspondence should be addressed to K. Jabeen; kanwaljabeen44@yahoo.com
}

Received 2 November 2020; Revised 21 January 2021; Accepted 8 February 2021; Published 19 February 2021

Academic Editor: Jerzy Baranowski

Copyright (C) 2021 R. M. Akram Muntazir et al. This is an open access article distributed under the Creative Commons Attribution License, which permits unrestricted use, distribution, and reproduction in any medium, provided the original work is properly cited.

\begin{abstract}
Due to potential implications, boundary layer analysis of chemically reacting Carreau nanofluid has been carried out to examine flow properties of ferromagnetic fluid over a stretched sheet in the presence of magnetic dipole, for shear thinning and shear thickening fluids. Furthermore, the transportation of heat under thermal radiation, heat generation, the Brownian, and thermophoresis aspects has been evaluated. The dimensionless form of highly nonlinear coupled partial differential equations is obtained using suitable similarity transformations and then solved numerically by well-known bvp4c technique via MATLAB based on the shooting method. The outcomes of physical quantities are presented through graphs and numerical benchmarks. Moreover, outcomes for skin fraction, Sherwood and Nusselt numbers for velocity, concentration, and temperature are also estimated in this study. The present study reveals that the concentration and thermal boundary layer thicknesses were higher for shear thinning $(n<1)$ fluid when compared with shear thickening $(n>1)$ fluids, but reverse effects are to be observed for momentum boundary layer thickness.
\end{abstract}

\section{Introduction}

Fast progresses in multidisciplinary approach in industrial, technological, and engineering developments claim the efficient and well-organized energy utilization and its implementations in a vast proportion. Consequently, nanofluids recently have one of the influential research themes amongst thermal technologies due to their diverse applications and thermal management. Nanoparticles are self-assembled in the presence of magnetic field in which magnetic movements tend to align the field direction in one dimension. So, the magnetic nanoparticles possess highly conductive paths for thermal flux and finally have a quick heat transport. Furthermore, a liquid strongly magnetized by magnetic field is named as ferrofluid. They are normally referred as magnetic fluids. In the presence of magnetic field, when magnetic nanoparticles are added to base fluid, they form a nanofluid subclass, as they frequently respond to applied magnetic field. These nanosized particles have an average size of $10 \mathrm{~nm}$. Although, for a stronger magnetic field ( $\gg 10 \mathrm{kG})$, a ferrofluid can possess its fluidity. The arrangements of elements in ferrofluids are most probably in the ratio as $85 \%$ liquid, $10 \%$ surfactant, and $5 \%$ magnetic solid by volume. The fluid flow by gravitational force or applied pressure gradient is common, but we need some extra energy to run such type of device to produce more pressure gradient. We need a fluid which can flow in situations without gravitational force or availability of energy stays for a long period. Ferrofluids are first modeled in early $60 \mathrm{~s}$ of 20th century and classified by Pappell [1] in 1963 at the National Aeronautics and Space Administration (NASA). He introduced the grinding procedure for ferrofluid. Later, ferrofluid's invention improved Pappell's procedure, and highly magnetic liquids were fabricated 
[1-3].There are basically three subclasses that state how material is influenced with magnetic field: (i) diamagnetism, very less influenced by the magnetic flux; (ii) paramagnetism, much more affected by field of force as compared to diamagnetism; and (iii) ferromagnetism, iron gadolinium, magnetite $\left(\mathrm{Fe}_{3} \mathrm{O}_{4}\right), \mathrm{CoFe}_{3} \mathrm{O}_{4}$, nickel, and $\mathrm{MnBi}$ are strenuously affected by the magnetic field and highly polarized in the magnetic field direction, and interestingly, they retain their state of polarization even when field is disconnected.

Collective behavior of all abovementioned types of fluids is referred as ferromagnetic fluids.

Ferrofluid has numerous practical applications within a scientific field. Some common examples are ferrofluid lubrication of bearings, sensors, hi-fi speakers, accelerometer, targeting cancer cells or tumors, magnetic-based devices, sensor applications, optical applications, biomedical engineering, and medicines.

Generally, fluids are categorized as Newtonian or nonNewtonian fluids which have a lot of industrial examples such as food industry, polymer industry, paper production, and many more extensive applications. For chemical engineering, the dominant property of fluids is non-Newtonian viscosity.

Fluids in which viscosity varies with shear rate are considered to be generalized Newtonian fluids. In such fluids, viscosity depends on the rate of shear. Up to two or three orders of magnitude change in viscosity may be convenient for some fluids but causes inconvenience for polymer processing and lubrication problems when considered. So, Newton's viscosity law was modified empirically and allowed the viscosity to change with rate of shear. This kind of fluid is commonly characterized as generalized Newtonian fluids [4].

The constitutive relation of power law is uncomplicated, generalized Newtonian fluid, but it has the deficiency that it cannot accurately predict viscosity for extremely high or low rates of shear. To overcome, another limitation model was considered, i.e., the Carreau rheological model (a combination of Newtonian and power law properties) proposed by Carreau [5], which confined the limitation of the power law model, and widely acceptable in technological processes and chemical engineering. For free surface flow, the fundamental relation of the Carreau model is suitable enough. The reason behind the fact is that, in the Carreau Model, when the shear rate approaches to zero, viscosity remains finite. This fluid possesses shear thinning $(n<1)$, shear thickening $(n>1)$, and Newtonian $(n=0)$ fluid properties [6]. Due to vast number of applications in the industrial, technological, and engineering developments, the Carreau model has received notable attention of researchers/authors in the last decades. Rosensweig and Neuringer [6] stated that a magnetic force per unit volume $\mu_{o}(M \cdot \nabla) H$ reduces to $\mu_{o} M \nabla H$ if the following conditions hold: (i) the magnetization $(M)$ is always in the magnetic field $(H)$ direction, (ii) the fluid must be nonconducting electrically, and (iii) the displacement current is insignificant.

Neuringer [7] treated the BLF along stagnation point and flat plate. Andersson [8] discussed a flow of ferrofluid with magnetic dipole. In this study, the authors mainly focus on the thermomechanical interaction with the magneto effect on heat transfer and skin friction. Convection characteristics of ferroliquid by heated cavity was explored by Kefayati [9] using the LBM scheme. Mixed convective and chemically reactive ferroliquid radiating flow was observed by Hayat et al. [10] under the effects of magnetic dipole. Zeeshan et al. [11-13] discussed the ferrofluid heat transfer over a stretchable sheet with magnetic field by the cause of magnetic dipole, prescriptive surface temperature, and heat flow.

In [14], Waqas studied the MHD Carreau fluid flow over an exponentially convected stretched sheet by computing thermophoresis and Brownian motion. They concluded that the velocity profile for local Weissenberg (We) number reduces in course of $n<1$, while it increases for $n>1$.

In [15], Carreau nanofluid problem composed of activation energy thermal expulsion with the parameters control method was investigated on a stagnation point, and Hsiao [15] claimed that the composed problem can be used to promote the system's economic efficiency. In [16], Khan et al. presented the solution of unsteady 2D Carreau nanofluid over a moving/static wedge by using bvp4c and shooting techniques. They had thoroughly discussed Brownian motion and thermophoresis effects and concluded that magnetic field parameter, unsteady parameter, and wedge angle greatly affected the thermal and concentration profiles in both cases when $n<1$ and $n>1$. They also noticed that fluid's velocity was shorter for fixed wedge as comparable with stretched wedge. Mathematical relation for MHD 3D Carreau nanofluid radiative flow was established by Irfan et al. [17] on a bidirectional stretching sheet, and the problem was tackled by bvp4c by supposing nanoparticle zero flux. They examined that the effects of We for $n<1$ and $n>1$ were completely reverse on thermal and concentration profiles.

2D MHD Carreau fluid [18, 19] analysis of gyrotactic microorganisms $[18,20]$ over a slendering sheet was investigated for variable thickness and slenderness parameters using Runge-Kutta-based Newton's scheme. Multiple slip effects were taken in account with Soret and Dufour effects for the controlling process of the mass and heat transferring and exerted the modifying and controlling influences on mass and heat transfer rate for distinct numbers of slendering parameters. Also, Soret and Dufour numbers are used to modulate the local Sherwood and Nusselt numbers.

Ferromagnetic 2-D Carreau nanofluid [21, 22] material flow by moving surface with effects of external magnetic field due to magnetic dipole [21-23] was modeled by Waqas [23], and he made comparison for $(n<1)$ and $(n>1)$ liquids considering Brownian and thermophoresis variables. Basha et al. [24] mainly addressed the flow of ferromagnetic Carreau nanofluid at a stagnation, wedge, and porous plate, with numerical techniques with effects of magnetic dipole for shear thinning/thickening $(n<1$ and $n>1)$ cases. The We number on shear thinning/thickening nanofluids has opposite impacts on velocity and thermal profiles, but suction/ injection leads to increase the fluid velocity. The temperature profile for shear thinning/thickening nanofluid increases for increasing values of thermophoretic and ferromagnetichydrodynamic interaction, but fluid concentration declines by raising Brownian motion. In short, they observed that the shear thinning fluid has slightly higher temperature than 
shear thickening fluid. The main findings for engineering aspects are as follows: in comparison of wedge, plate, and at the stagnation point, the flow past of a plate has less skin friction and low heat transfer rate for both $n<1$ and $n>1$ cases. A study of nonlinear thermally radiating Carreau nanofluid flow along magnetic dipole effects by shooting technique was explored by Waqas et al. [25, 26].

Iqbal [27], Taseer [28], and Mishra [29] addressed 2D and 3D flow of MHD Carreau nanofluid with chemical reaction over an exponential surface and presented detailed discussion upon physical outcomes of various parameters such as Weissenberg number, thermal and concentration Biot numbers, Brownian movement thermophoresis, Schmidt and Prandtl numbers, thermal radiation and temperature ratio parameter for primary and secondary velocities, concentration and temperature, and for both shear thickening $(n=1.5)$ and shear thinning $(n=0.5)$ flows [30-32].

Bhatti [33] explored the heat transfer on the electromagnetohydrodynamic (EMHD) Carreau fluid flow over rectangular plates. For physical modeling, he considered a Darcy-Brinkman-Forchheimer medium and solved the formulated flow system by numerical and semianalytical schemes. In his work, the author observed the Forchheimer and MF suppress the flow. Also, he concluded that the We and $n$ retard the velocity of fluid but enhance the temperature profile. He declared that DTM is more powerful technique [34, 35]. In the study [36], Arain et al. studied the flow behavior of Carreau fluid along rotating circular plates with gyrotactic microorganisms under the effects of magnetic generalized Reynolds \# and concluded that fluid viscosity declines in shear thinning case when comparing with Newtonian and shear thickening fluids.

A chemical reaction is a process in which a chemical substance deforms from one form to another form by changing electrons' positions and makes a bond among atoms. Chemical reactions, in chemical engineering processes, are basically used to generate new compounds by raw materials. Some common examples to discuss the fluid flows with chemical reaction are extraction of petroleum from mineral ores, fibrous insulation, water pollutions, the dispersion and formation of fog, and production of ceramics.

Due to potential implications and applications, boundary layer analysis of chemically reacting Carreau nanofluid to examine flow properties of ferromagnetic fluid over a stretched sheet in the presence of magnetic dipole, heat generation, the Brownian, thermophoresis motion, viscous, and ohmic dissipation for shear thinning and shear thickening fluids has been carried out. The problem is solved by numerical technique bvp4c [30-32]. Magnetized Carreau nanofluids are used in diverse applications, for example, malice usage, aeronautics zones, mechanical mechanisms, therapeutic diligences, micro microelectronic, and thermonuclear depots. These reforms can be acknowledged and applied as projecting forthcoming of viable energy strategies for the progression.

\section{Formulation of the Flow Model}

Two-dimensional ferromagnetic Carreau (generalized Newtonian) nanofluid flow over an impermeable stretched sheet placed horizontally under the effects of the external magnetic field is shown in Figure 1. Opposite but two equal forces are applied along the $x$ direction, while $y$-axis is normal to the flow.

A magnitude dipole having its center at distance "a" on $y$-axis against the sheet is located. Magnetic field due to dipole towards $x$ direction (positive) rises the magnetic field to saturate the ferrofluid. Temperature of the sheet $T_{w}$ is kept under the Curie temperature $T_{c}$ (above the situation/temperature, ferromagnetic nanoparticles lose their ferromagnetism and become paramagnetic). Whereas, the elements of fluid referring to the sheet are supposed having temperature $T_{\infty}=T_{c}$. So, they are ineffectual to magnetize till they start to cool while penetrating in thermal boundary layer region.

The structure of nonlinear thermal radiation, viscous dissipation with chemically reacting fluids under the magnetic dipole effects upon generalized Newtonian (Carreau fluid) nanofluid, is modeled. The governing boundary layer equations are as follows:

$$
\begin{array}{r}
u \frac{\partial u}{\partial x}+v \frac{\partial u}{\partial y}=v \frac{\partial^{2} u}{\partial y^{2}}\left[1+\Gamma^{2}\left(\frac{\partial u}{\partial y}\right)^{2}\right]^{((n-1) / 2)}+v(n-1) \Gamma^{2} \frac{\partial^{2} u}{\partial y^{2}}\left(\frac{\partial u}{\partial y}\right)^{2}\left[1+\Gamma^{2}\left(\frac{\partial u}{\partial y}\right)^{2}\right]^{((n-3) / 2)}+\frac{\lambda_{0} M}{\rho} \frac{\partial H}{\partial x}, \\
u \frac{\partial T}{\partial x}+v \frac{\partial T}{\partial y}+\frac{\lambda_{0}}{\rho C_{p}} T \frac{\partial M}{\partial T}\left(u \frac{\partial H}{\partial x}+v \frac{\partial H}{\partial y}\right)=\frac{k}{\rho C_{p}} \frac{\partial^{2} T}{\partial y^{2}}+\frac{16 \sigma^{*}}{3 m^{*} \rho C_{p}} \frac{\partial}{\partial y}\left(T_{c}^{3} \frac{\partial T}{\partial y}\right) \\
+\frac{Q^{*}}{\left(\rho C_{p}\right)}\left(T-T_{c}\right)+\frac{\nu}{C_{p}}\left(\frac{\partial u}{\partial y}\right)^{2}\left[1+\Gamma^{2}\left(\frac{\partial u}{\partial y}\right)^{2}\right]^{((n-1) / 2)}+\tau\left[D_{B} \frac{\partial C}{\partial y} \frac{\partial T}{\partial y}+\frac{D_{T}}{T_{c}}\left(\frac{\partial T}{\partial y}\right)^{2}\right], \\
u \frac{\partial C}{\partial x}+v \frac{\partial C}{\partial y}=D_{B} \frac{\partial^{2} C}{\partial y^{2}}+\frac{D_{T}}{T_{c}} \frac{\partial^{2} T}{\partial y^{2}}-k_{r}\left(C-C_{c}\right),
\end{array}
$$




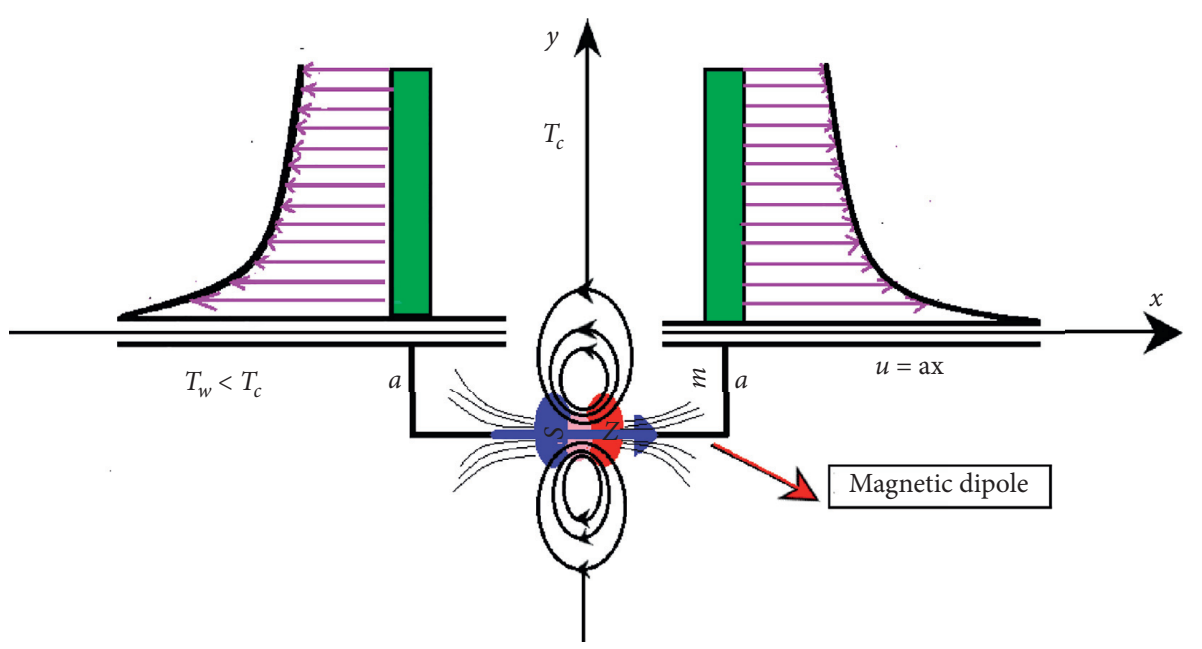

Figure 1: Physical interpretation of the flow model.

where $v$ is the kinematics viscosity, $\lambda_{o}$ is the magnetic permeability, $C_{p}$ is the specific heat, $n$ is the power law index, $H$ is the magnetic field, $M$ is the magnetization, $Q^{*}$ is the coefficient of heat generation/absorption, and $D_{B}$ and $D_{T}$ are the coefficients of Brownian and thermophoretic movement.

2.1. Boundary Conditions. The related Bc's are given as follows:

$$
\left\{\begin{array}{l}
\mathbf{y}=0: \\
u=a x, v=0, T=T_{w}, C=C_{w}, \\
\mathbf{y} \longrightarrow \infty: \\
u \longrightarrow 0, T \longrightarrow T_{c}, C \longrightarrow C_{c} .
\end{array}\right.
$$

Here, $a$ indicates the rate of stretching. It was observed that two forces with equal magnitude but opposing directions are applied upon the sheet while keeping a constant origin. Also, $u=\left.a x\right|_{y=0}$ is the stretching velocity of the Carreau nanofluid, and $v=\left.0\right|_{y=0}$ represents the surface has zero suction or injection, i.e., the sheet is impermeable. Furthermore, $T=\left.T_{w}\right|_{y=0}$ and $C=\left.C_{w}\right|_{y=0}$ show wall temperature and concentration. Whereas, $\left.u \longrightarrow \infty\right|_{y \rightarrow \infty}$ indicates that velocity of free stream is zero. While, $\left.T \longrightarrow T_{c}\right|_{y \longrightarrow \infty}$ and $\left.C \longrightarrow C_{c}\right|_{y \longrightarrow \infty}$ illustrate the ambient fluid temperature and concentration.

2.2. Magnetic Dipole Expression. The ferrofluid flow is affected via the magnetic field $(\mathrm{H})$ subjected to magnetic dipole, whose magnetic scalar potential $(\Phi)$ is as follows:

$$
\Phi=\frac{\gamma}{2 \pi}\left(\frac{x}{x^{2}+(y+a)^{2}}\right)
$$

where " $\gamma$ " represents the strength of the magnetic field, and $H_{x}$ and $H_{y}$ in $x$-axis and $y$-axis directions are the magnetic field components:

$$
\begin{aligned}
& H_{x}=-\frac{\partial \Phi}{\partial x}=\frac{\gamma}{2 \pi}\left[\frac{x^{2}-(y+a)^{2}}{\left(x^{2}+(y+a)^{2}\right)^{2}}\right], \\
& H_{y}=-\frac{\partial \Phi}{\partial y}=\frac{\gamma}{2 \pi}\left[\frac{2 x(y+a)}{\left(x^{2}+(y+a)^{2}\right)^{2}}\right] .
\end{aligned}
$$

The intensity of $H$ is

$$
H=\left[\left(\frac{\partial \Phi}{\partial x}\right)^{2}+\left(\frac{\partial \Phi}{\partial y}\right)^{2}\right]^{(1 / 2)}
$$

From above expression, one can obtain

$$
\frac{\partial H}{\partial x}=-\frac{\gamma}{2 \pi}\left(\frac{2 x}{(y+a)^{4}}, \frac{\partial H}{\partial y}=\frac{\gamma}{2 \pi}\left(\frac{-2}{(y+a)^{3}}+\frac{4 x^{2}}{(y+a)^{5}}\right)\right. \text {, }
$$

where $H$ is supposed to be strong enough for saturating the ferromagnetic fluid, and $M$ via temperature in the linear state relation can be approximated as

$$
M=K\left(T_{c}-T\right),
$$

where constant $K$ is the pyromagnetic coefficient. Also, ferrohydrodynamic interaction needs that the fluid is at a state when $T$ is less than $T_{c}$, i.e., $\left(T<T_{c}\right)$, and secondly applied magnetic field must be nonhomogeneous. So that, when ferrofluid attains $T_{c}$, no additional magnetization will appear. So, as shown in equation (7), the ferroliquid will never be considered away from the sheet at $T_{c}$. 
2.3. Similarity Transformations. Introducing the similarity transformations $[11,23]$,

$$
\left.\begin{array}{ll}
\eta=\sqrt{\frac{a}{v}} y, & \psi=x f(\eta) \sqrt{a \nu} \\
u=a x f^{\prime}(\eta), & v=-\sqrt{a \nu} f \\
\theta(\eta)=\frac{T_{c}-T}{T_{c}-T_{w}}, & \phi(\eta)=\frac{C_{c}-C}{C_{c}-C_{w}}
\end{array}\right\} .
$$

2.4. Transformed Model. We have the following transformed system:

$$
\begin{array}{r}
\left(1+\mathrm{We}^{2} f^{\prime \prime^{2}}\right)^{(n-3 / 2)}\left(1+n \mathrm{We} \mathrm{e}^{2} f^{\prime \prime 2}\right) f^{\prime \prime \prime}+f f^{\prime \prime}-f^{\prime 2}-\frac{2 \beta \theta}{(\eta+\alpha)^{4}}=0, \\
(1+R) \theta^{\prime \prime}+\operatorname{Pr}\left(f \theta^{\prime}+N b \theta^{\prime} \phi^{\prime}+N t \theta^{\prime 2}-E c \beta(\theta-\varepsilon)\left(\frac{2 f^{\prime}}{(\eta+\alpha)^{4}}+\frac{4 f}{(\eta+\alpha)^{5}}\right)+S \theta+E c f^{\prime \prime 2}\left(1+W e^{2} f^{\prime \prime 2}\right)^{(n-1 / 2)}\right) \\
+\lambda \beta(\theta-\varepsilon)\left(\frac{2 f}{(\eta+\alpha)^{3}}=0, \phi^{\prime \prime}+S c f \phi^{\prime}+\left(\frac{N t}{N b}\right) \theta^{\prime \prime}-S c K_{r} \phi=0 .\right.
\end{array}
$$

Also, the transformed BC's are

$$
\left\{\begin{array}{l}
f(0)=0 ; \\
f^{\prime}(0)=1 ; \\
f^{\prime}(\infty)=0 ; \\
\theta(0)=1 ; \\
\theta(\infty)=0 ; \\
\phi(0)=1 ; \\
\phi(\infty)=0 .
\end{array}\right.
$$

Here, $\mathrm{We}^{2}=\left(\Gamma^{2} x^{2} a^{3} / \nu\right)$ is the Weissenberg number, $\alpha=$ $\sqrt{\left(a a_{o}^{2} / \nu\right)}$ is the dimensionless distance, $\beta=\left(\lambda_{o} \rho \gamma / 2 \pi \mu^{2}\right) K\left(T_{c}-T_{w}\right)$ is the ferrohydrodynamic interaction parameter, $\operatorname{Pr}=\left(\mu C_{p} / k\right)$ is the Prandtl number, $S=\left(Q^{*} / a \rho C_{p}\right)$ is the heat generation parameter, $R=\left(16 \sigma^{*} T_{c}^{3} / 3 \mathrm{~km}^{*}\right)$ is the thermal radiation, $\varepsilon=\left(T_{c} / T_{c}-\right.$ $\left.T_{w}\right)$ is the curie temperature, $E c=\left(a^{2} x^{2} / C_{p}\left(T_{w}-T_{c}\right)\right)$ is the viscous dissipation, $\lambda=\left(a \mu^{2} / \rho k\left(T_{w}-T_{c}\right)\right)$ is the temperature ratio, $\mathrm{Nb}=\left(\tau D_{B}\left(C_{w}-C_{c}\right) / \nu\right)$ is the Brownian movement parameter, $\mathrm{Nt}=\left(\tau D_{T}\left(T_{w}-T_{c}\right) / T_{c} \nu\right)$ is the thermophoretic movement parameter, $\mathrm{Sc}=\left(\nu / D_{B}\right)$ is the Schmidt number, and $K_{r}=\left(k_{r} / a\right)$ is the chemical reaction parameter.

2.4.1. Physical Quantities. The significant physical parameters of engineering concerns are dimensionless Sherwood number $\mathrm{Sh}_{x}$, Nusselt numbers $N_{u x}$, and skin friction $C_{f x}$ that physically indicates rate of mass/heat transfers and shear stress, respectively, and are defined as

$$
\begin{aligned}
C_{\mathrm{fx}} & =\frac{\tau_{w}}{\rho U^{2}}, \\
N_{\mathrm{ux}} & =\frac{x q_{w}}{k\left(T_{c}-T_{w}\right)}, \\
\mathrm{Sh}_{x} & =\frac{x q_{s}}{D_{B}\left(C_{c}-C_{w}\right)},
\end{aligned}
$$

where $\tau_{w x}$ is the shear stress at the sheet along $x$-axis: $\tau_{w}=\left[\mu_{o} a f^{\prime \prime}(0)\left(1+W e^{2}\left(f^{\prime \prime}(0)\right)^{2}\right)^{(n-1 / 2)}\left(\operatorname{Re}_{x}\right)^{(1 / 2)}\right]_{y=0} ; \quad q_{w}$ and $q_{s}$ are the surface heat and mass fluxes defined as $q_{w}=-k(\partial T / \partial y)_{y=0}-\left(\left(16 \sigma^{*} / 3 k^{*}\right)\left(T_{c}^{3}(\partial T / \partial y)\right)\right)_{y=0}$, $q_{s}=-D_{B}(\partial C / \partial y)_{y=0}$. Upon fixing the local Reynold number $\operatorname{Re}_{x}=(a x / \nu)$ and using the above defined relations, we have $\left(\operatorname{Re}_{x}\right)^{(1 / 2)} C_{f x}=f^{\prime \prime}(0)\left(1+W e^{2}\left(f^{\prime \prime}(0)\right)^{2}\right)^{(n-1 / 2)}$

$\left(\operatorname{Re}_{x}\right)^{(-1 / 2)} N_{u x}=(1+R) \theta^{\prime}(0)\left(\operatorname{Re}_{x}\right)^{(-1 / 2)} \mathrm{Sh}_{x}=-\phi^{\prime}(0)$.

\section{Results and Discussion}

The current study reveals that the governing boundary layer equations for Carreau nanofluid over a stretching sheet are coupled and highly nonlinear, and due to extreme difficulty regarding the complex nonlinearity, the exact solution for the governing nonlinear problem cannot be obtained. This situation can be tackled numerically, and hence, numerical solution for the transformed system under the boundary conditions is computed by MATLAB solver bvp4c [30-32] for various substantial physical parameters. Salient features of significant parameters are We (Weissenberg number), Pr and Ec (Prandtl and Eckret numbers), R (thermal radiation parameter), $\mathrm{Nt}, \mathrm{Nb}$ (thermophoresis and Brownian motion parameters), Sc (Schmidt number), S (heat generation/ 
TABLE 1: Calculation of velocity, thermal, and solutal gradients of Carreau nanofluid for different values of Weissenberg parameters.

\begin{tabular}{lccccc}
\hline & \multicolumn{2}{c}{$\left(\mathrm{Re}_{x}\right)^{(1 / 2)} C_{f x}$} & \multicolumn{2}{c}{$\left(\mathrm{Re}_{x}\right)^{(-1 / 2)} N_{u x}$} & \multicolumn{2}{c}{$\left(\mathrm{Re}_{x}\right)^{(-1 / 2)} \mathrm{Sh}_{x}$} \\
We & $n=0.5$ & $n=1.5$ & $n=0.5$ & $n=1.5$ & $n=0.5$ \\
\hline 1.2 & 1.660117 & 1.992135 & 0.356374 & 0.494855 & 0.835744 \\
1.8 & 1.551527 & 2.053859 & 0.287481 & 0.514654 & 0.766914 \\
2.4 & 1.477283 & 2.106286 & 0.206023 & 0.530434 & 0.685165 \\
3.5 & 1.370560 & 2.185403 & 0.123823 & 0.552645 & 0.964538 \\
\hline
\end{tabular}

absorption), and $n$ (index of power law). Here, both $(n>1)$ and $(n<1)$ cases are elaborated in figures and tables. For the present computational work, the pertinent values throughout the analysis are taken as $\alpha=1, \beta=2$, We $=1, \operatorname{Pr}=1.5, N b=0.1, N t=0.1, S=0.1, E c=0.2, \varepsilon=0.1$, $R=2, \lambda=0.1, S c=2, K_{r}=0.1$. Moreover, the results for the skin friction, Nusselt and Sherwood numbers, are tabulated (1-3) and deliberated in details.

Table 1 represents the analysis of velocity, thermal, and solutal gradient of Carreau nanofluid for distinct values of Weissenberg parameter, both shear thickening $(n>1)$ and shear thinning $(n<1)$ cases. Clearly, for shear thinning $(n=0.5)$, liquid $\quad\left(\operatorname{Re}_{x}\right)^{(1 / 2)} C_{f x}, \quad\left(\operatorname{Re}_{x}\right)^{(-1 / 2)} N_{u x}$, and $\left(\operatorname{Re}_{x}\right)^{(-1 / 2)} \operatorname{Sh}_{x}$ decreases, while it increases for $n=1.5$. Tables 2 and 3 show the computed results of $\left(\operatorname{Re}_{x}\right)^{(-1 / 2)} N_{u x}$ and $\left(\operatorname{Re}_{x}\right)^{(-1 / 2)} \mathrm{Sh}_{x}$ for different parameters.

Figure 2 demonstrates the behavior of We on velocity, thermal, and concentration profiles for $n=0.5$ and $n=1.5$. It was noticed that for varying values of We, the velocity of the fluid declines in the case when $n<1$, but it increases for increasing values of We when $n>1$. Actually, We is the relation between relaxation time and the process in which time grows the fluid's viscosity. Increasing the values of We enhances the relaxation time. So, there is a decrement in the fluid's velocity due to dominating effects of viscosity; this generates resistance in the fluid particles. Moreover, the plots of the variation in the thermal and solutal distributions for various values of We can also be seen in Figures 2(b) and 2(c) for both types of fluids. These figures exhibit that the thermal and concentration layer thickness increases for increasing We in shear thinning case. Scientifically, ratio of elastic and viscous forces is known as We, so it elevates the thickness of fluid so temperature increases. While, both profiles show decreasing trend for shear thickening fluids. It is necessary to mention here that $\mathrm{We}=0$ is the recovered Newtonian fluid.

Figure 3 visualizes the impacts of $\beta$ which is a parameter of ferromagnetic interaction. $\beta$ demonstrates effects of applied external magnetic field induced by magnetic dipole. The velocity profile (Figure 3(a)) decreases for both cases as $\beta$ increases. As a fact, the magnetic field reacts as an opposing force during the flow when $\beta$ gets higher. This takes place because the interaction results in a drag force cause to reduce flow velocity. The temperature boundary layer increases for both cases as shown in Figure 3(b). In fact, there is an intervention between the applied magnetic field and motion of the fluid; this causes reduction in velocity and augmented the frictional heating among the fluid layers; this produces
TABLE 2: Calculation of thermal gradient of Carreau fluid for different values of auxiliary parameters.

\begin{tabular}{|c|c|c|c|c|c|c|}
\hline \multirow[b]{3}{*}{$\operatorname{Pr}$} & \multirow[b]{3}{*}{$\mathrm{Ec}$} & \multirow[b]{3}{*}{$\mathrm{R}$} & \multirow[b]{3}{*}{$\mathrm{Nb}$} & \multirow[b]{3}{*}{$\mathrm{Nt}$} & \multicolumn{2}{|c|}{$\left(\operatorname{Re}_{x}\right)^{(-1 / 2)} N_{u x}$} \\
\hline & & & & & $n=0.5$ & $n=1.5$ \\
\hline & & & & & & \\
\hline 1.0 & & & & & 0.341029 & 0.487104 \\
\hline 1.2 & & & & & 0.352394 & 0.496773 \\
\hline 1.4 & & & & & 0.364992 & 0.507309 \\
\hline 1.6 & & & & & 0.378877 & 0.518403 \\
\hline & 0.1 & & & & 0.417960 & 0.628846 \\
\hline & 0.3 & & & & 0.398120 & 0.558445 \\
\hline & 0.5 & & & & 0.385222 & 0.510990 \\
\hline & 0.8 & & & & 0.372604 & 0.463111 \\
\hline & & 0.9 & & & 0.378877 & 0.499413 \\
\hline & & 1.2 & & & 0.363549 & 0.493744 \\
\hline & & 1.6 & & & 0.344012 & 0.489377 \\
\hline & & 2.0 & & & 0.325551 & 0.487104 \\
\hline & & & 0.8 & & 0.406514 & 0.518051 \\
\hline & & & 0.9 & & 0.392502 & 0.502367 \\
\hline & & & 1.0 & & 0.378877 & 0.487104 \\
\hline & & & 1.2 & & 0.352749 & 0.457801 \\
\hline & & & & 0.1 & 0.378877 & 0.487104 \\
\hline & & & & 0.3 & 0.360925 & 0.468066 \\
\hline & & & & 0.5 & 0.343564 & 0.449666 \\
\hline & & & & 0.7 & 0.326768 & 0.431877 \\
\hline
\end{tabular}

TABle 3: Solutal gradient for different values of auxiliary parameters.

\begin{tabular}{lccc}
\hline & & \multicolumn{2}{c}{$\left(\mathrm{Re}_{x}\right)^{(-1 / 2)} \mathrm{Sh}_{x}$} \\
Sc & $K_{r}$ & $n=0.5$ & $n=1.5$ \\
\hline 1.4 & & -0.686293 & -0.768921 \\
1.8 & -0.803091 & -0.898063 \\
2.0 & & -0.857703 & -0.957792 \\
2.2 & & -0.910142 & -1.014964 \\
\hline & 0.1 & -0.857702 & -0.957792 \\
& 0.3 & -1.083401 & -1.157533 \\
& 0.5 & -1.265232 & -1.325413 \\
& 0.7 & -1.421543 & -1.473035 \\
\hline
\end{tabular}

enhancement in thermal profile. Figure 3(c) depicts the behavior of $\beta$. The concentration profile increases for both cases $n<1$ and $n>1$.

Figure 4 reflects the effect of $S$ on $\theta(\eta)$ for both cases $n<1$ and $n>1$. It is evident from this figure that temperature profile accelerates for enhancing parameter $S$. Physically, more heat is generated in the system for $S$, and therefore, an improvement in temperature occurs. 


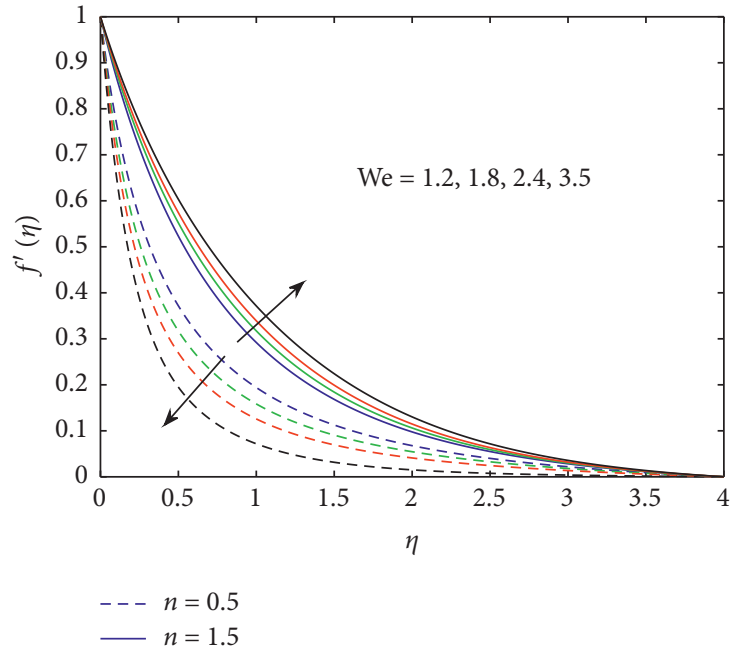

(a)

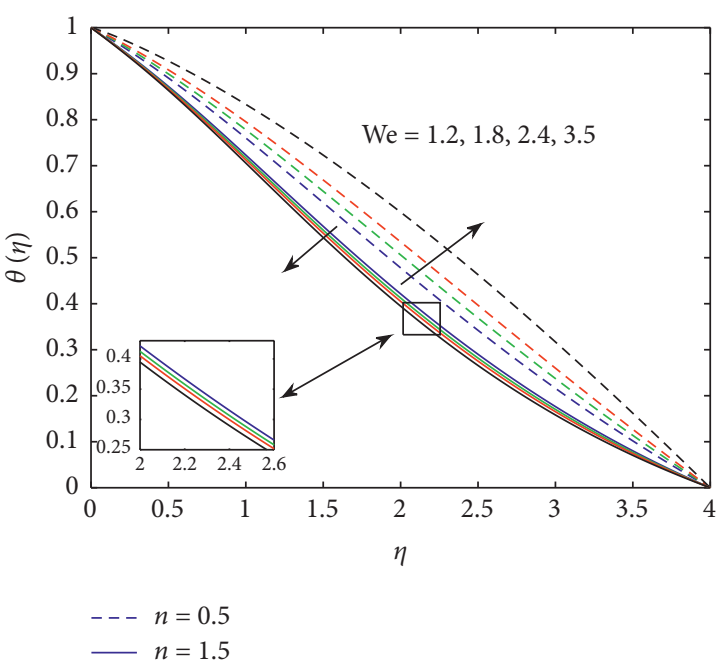

(b)

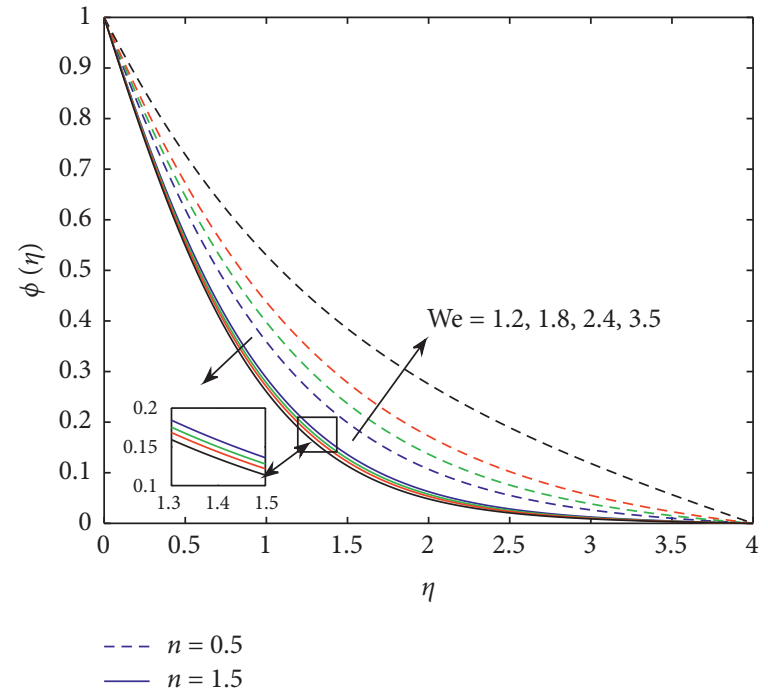

(c)

Figure 2: We effects on $f^{\prime}(\eta), \theta(\eta)$, and $\phi(\eta)$.
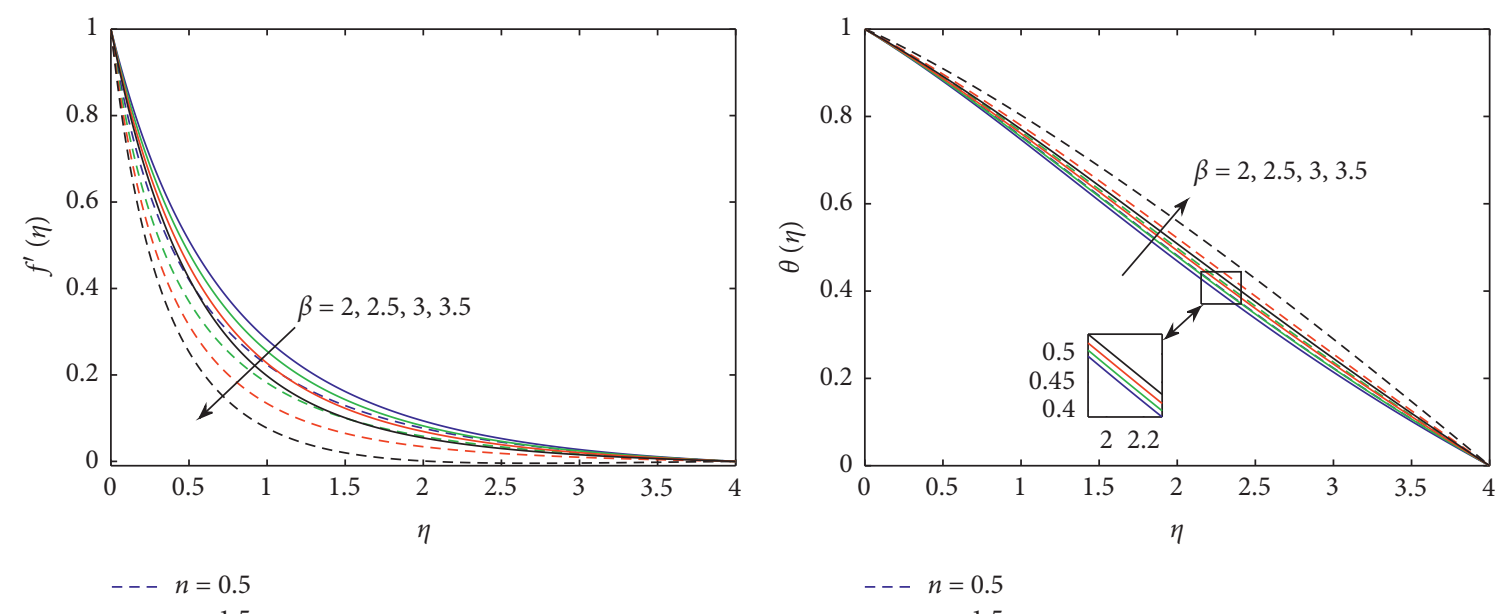

(a)

Figure 3: Continued. 


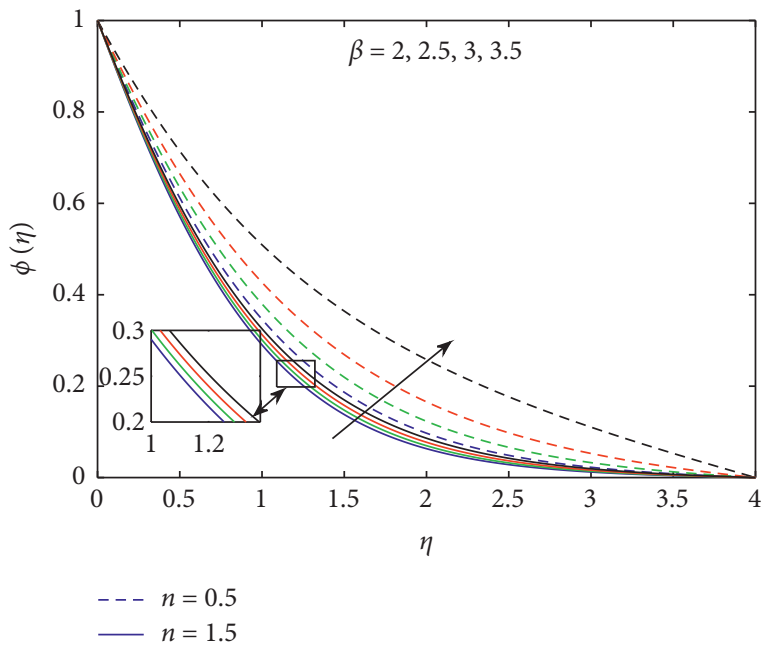

(c)

Figure 3: $\beta$ effects on $f^{\prime}(\eta), \theta(\eta)$, and $\phi(\eta)$.

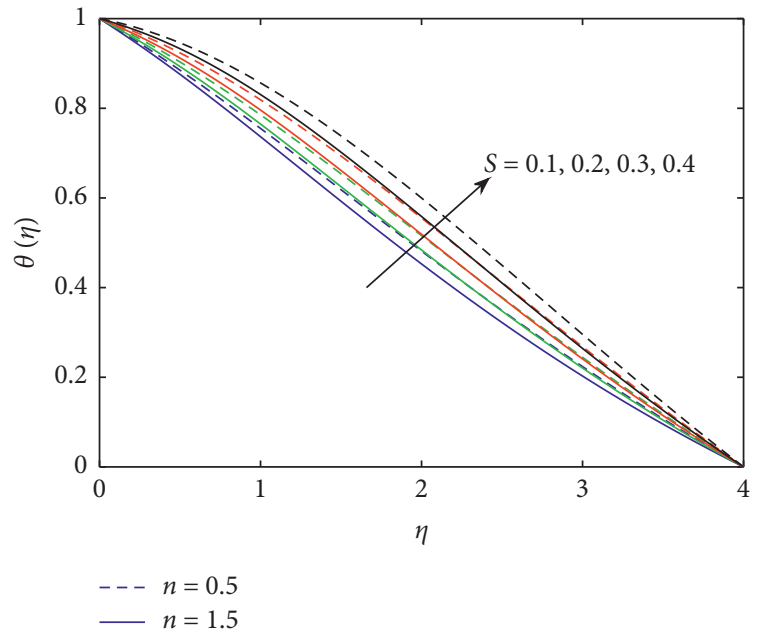

(a)

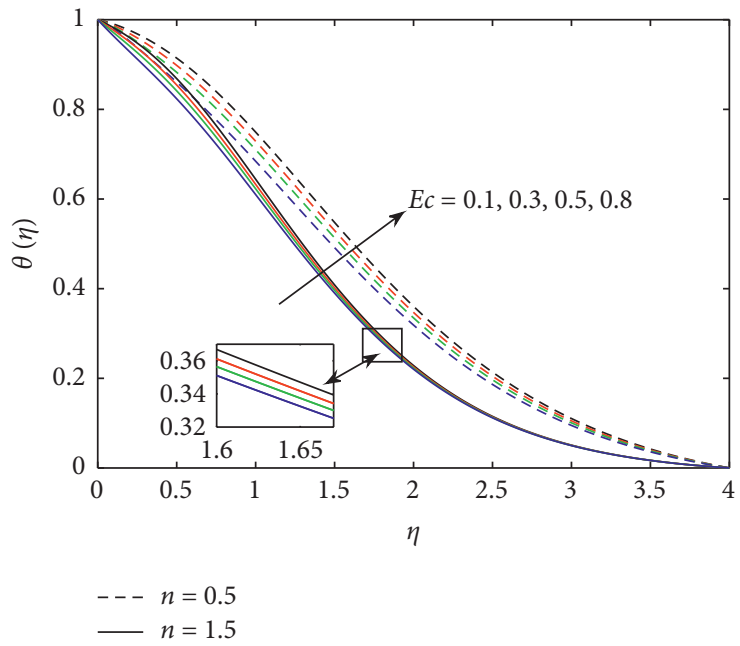

(b)

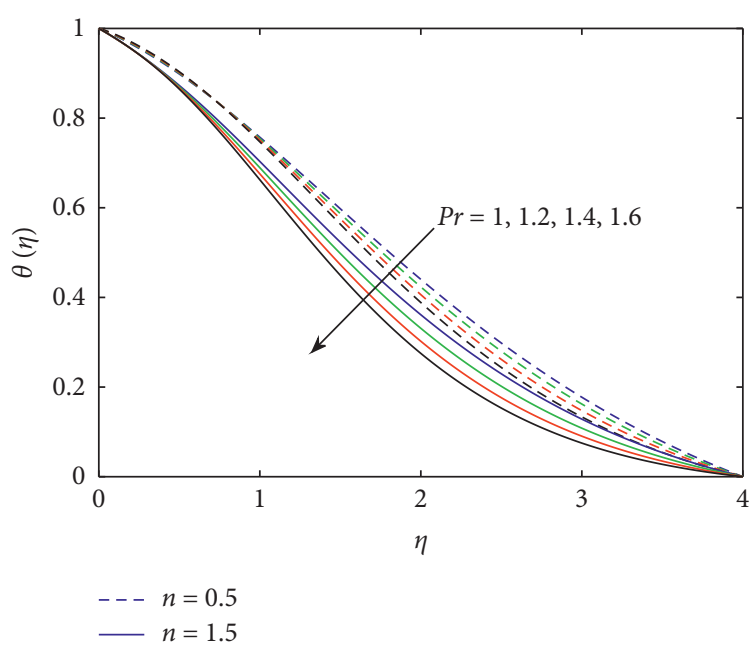

(c)

Figure 4: Effects of $S$, Ec, and $\operatorname{Pr}$ on $\theta(\eta)$. 


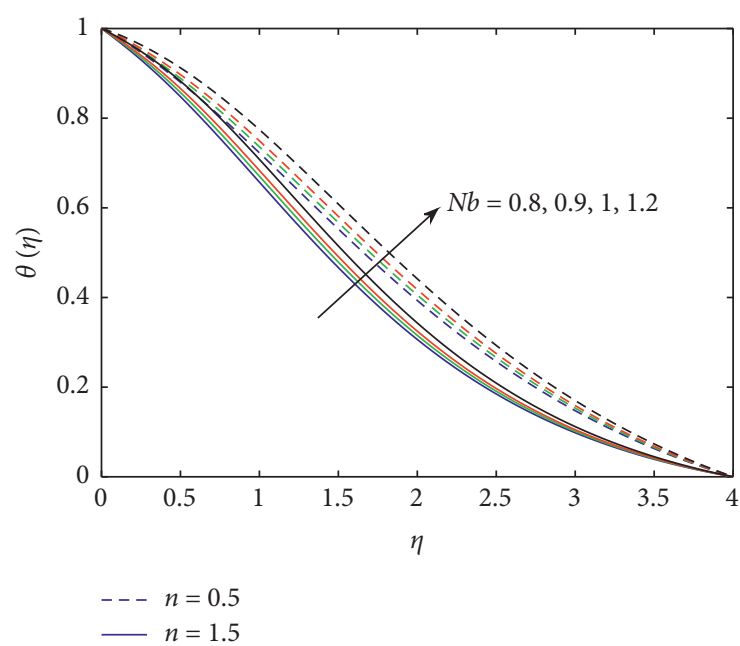

(a)

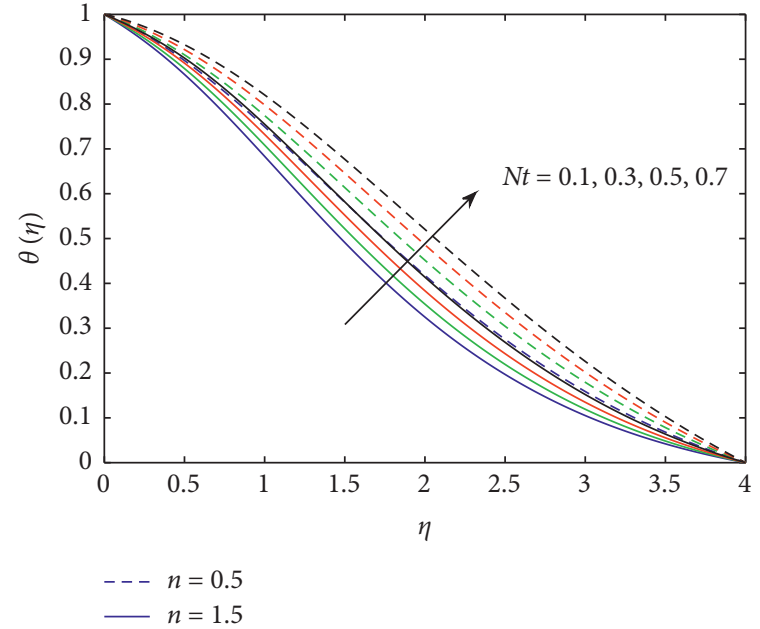

(b)

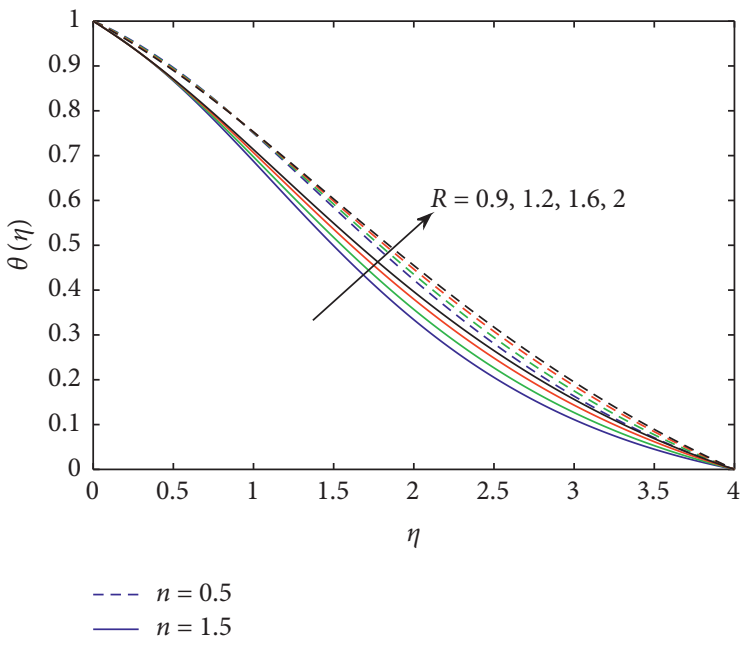

(c)

Figure 5: Effects of $\mathrm{Nb}, \mathrm{Nt}$ and $\mathrm{R}$ on $\Theta(\mu)$.

Viscous dissipation effect Ec is incorporated in Figure 4(b), and accelerated trend in the thermal boundary layer is observed in the flow region for higher values of Ec number for both situations.

Behavior of Prandtl number is illustrated by Figure 4(c) with both fluids. It is seen that increasing $\operatorname{Pr}$ causes deceleration in the thermal profile. Pr is the ratio between momentum diffusivity to thermal diffusion. So, when the Pr gets smaller, the thermal boundary layer thickness becomes larger. Hence, Pr may be used to increase the rate of cooling in conducting fluid flows.

Figure 5 highlights the impacts of $\mathrm{Nb}$ and $\mathrm{Nt}$ (Brownian and thermophoresis parameter). Both parameters show escalating trend on thermal profile for both $n=0.5$ and $n=1.5$ cases for increasing values of $\mathrm{Nb}$ and Nt nanoparticles. These nanoparticles show a powerful impact of heat transport properties of Carreau fluid. It is observed that with the increase in the value of $\mathrm{Nb}$, the temperature of nanoparticles enhanced. Physically, the reason behind the fact is the Brownian diffusion being random motion of nanoparticles suspended in fluid; this random motion intensity boosts the kinetic energy of the nanoparticles and results in overall increase in nanomaterial temperature. Furthermore, in Figure 5(b), the thermophoresis phenomenon, the nanoparticles affected by temperature are dragged away from the hot to cold surface so the fluid temperature rises. Consequently, large amount of nanoparticles is moved away from the sheet which accelerates the temperature of Carreau liquid for both circumstances.

It is observed from Figure 5(c) that the radiation parameter $R$ enhances the temperature profile when the values of $R$ increase for both shear thinning and shear thickening fluids. Scientifically, the heat energy generates towards the flow when the radiation effects exceeds; these causes elevate the heat, and hence, temperature profile gets higher.

Figure 6 shows the effects of Schmidt number (Sc). As $\mathrm{Sc}$ increases, the concentration profile decreases. The above behavior of concentration profile is according to the existing physical situations. On explanatory note, the 


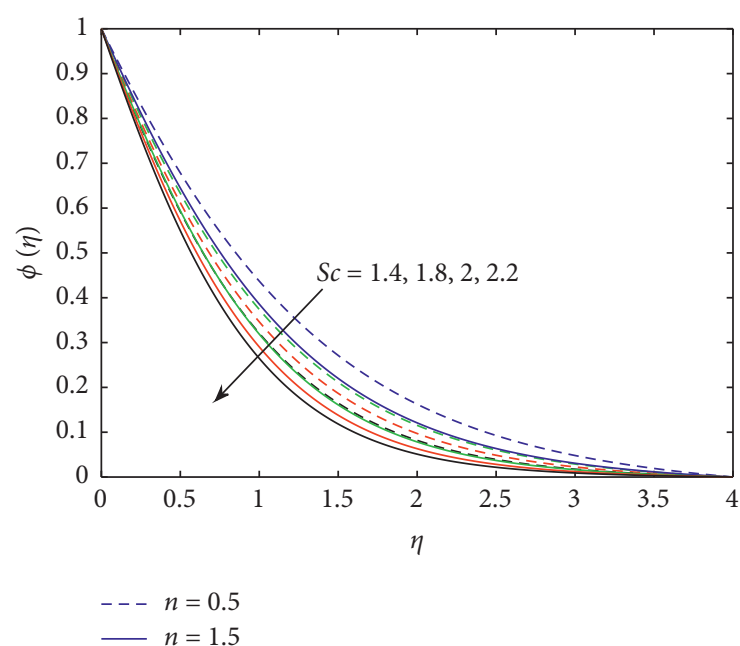

(a)

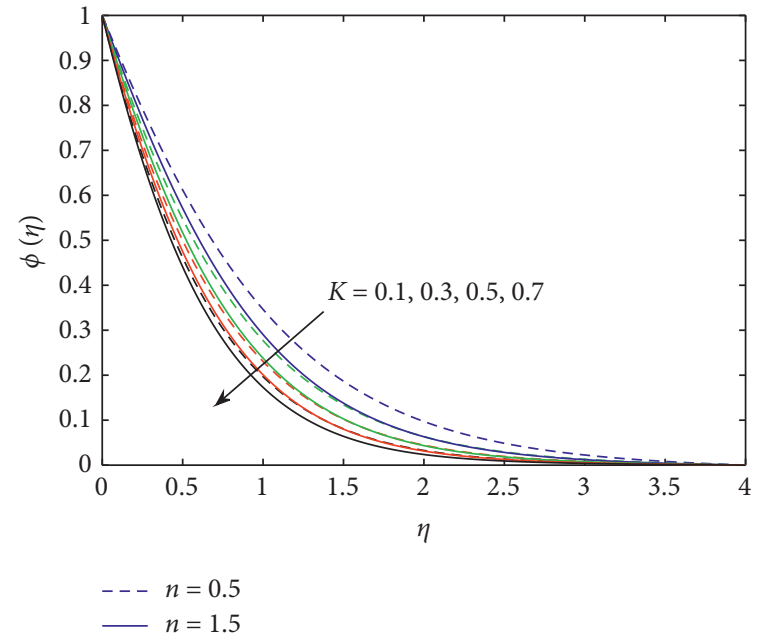

(b)

Figure 6: Effects of Sc and $\mathrm{K}$ on $\phi(\eta)$.

rate of mass transfer gets smaller when diffusion coefficient increases, and this retards the concentration profile for both cases. Similarly, higher molecular motion leads the sharpen process of mass transportation which resulted in the deceleration in the concentration profile. The graphical representation of chemical reaction parameter $K_{r}$ is shown in Figure 6(b). It is obvious from the figure that the concentration profile is decreasing rapidly for increasing values of $K_{r}$. This agrees with the physical reality that the high molecular motion leads the higher mass transmission process and in results, retards the fluid concentration profile.

\section{Conclusion}

With an expanding universal race, industrial and engineering developments require efficient energy utilization and its implementation. For this purpose, ferromagnetic Carreau (generalized Newtonian) nanofluid flow was considered under various parameters and external magnetic field. The keypoints of the current study are as follows:

(i) Increasing values of We accelerate $f^{\prime}(\eta)$ for shear thickening and decelerate for shear thinning case but reverse effects being observed for $\phi(\eta)$ and $\theta(\eta)$

(ii) Increasing values of $\beta$ decrease the fluid velocity but increase thermal and concentration profiles in both shear thickening and shear thinning cases

(iii) For increasing values, $\mathrm{S}, \mathrm{Ec}, \mathrm{Nt}, \mathrm{Nb}$, and $R \theta(\eta)$ increase but decrease for $\operatorname{Pr}$ in both cases $n<1$ and $n>1$

(iv) The concentration profile depreciate for the increasing values of Sc and $\mathrm{Kr}$ in both situations

(v) Skin friction coefficient, Nusselt, and Sherwood numbers are decreasing for shear thinning fluid but opposite behavior is seen for shear thickening fluid (vi) Shear thinning fluid has higher temperature than shear thickening fluid

\section{List of Symbols}

$u, v: \quad$ Velocity components

$\sigma: \quad$ Electrical conductivity

$\mathrm{x}, \mathrm{y}: \quad$ Space components

$v: \quad$ Kinematic viscosity

$n$ : $\quad$ Power law exponent

$\varepsilon: \quad$ Curie temperature

We: Weissenberg number $\alpha$ dimensionless distance

$D_{B}: \quad$ Brownian movement

$D_{T}: \quad$ Thermophoretic movement

$\lambda_{o}$ : $\quad$ Magnetic permeability

$H$ : $\quad$ Magnetic field

$Q^{*}: \quad$ Coefficient of heat generation

$\Phi: \quad$ Magnetic scalar potential

$K_{r}: \quad$ Chemical reaction parameter

$K: \quad$ Pyromagnetic coefficient

$\gamma: \quad$ Strength of magnetic field

$T_{c}: \quad$ Curie temperature

$T_{w}, C_{w}:$ Wall temperature and concentration.

\section{Data Availability}

The datasets used to support the results and conclusions of this study are included within this article.

\section{Conflicts of Interest}

The authors declare that they have no conflicts of interest.

\section{References}

[1] S. S. Papell, "Low viscosity magnetic fluid obtained by the colloidal suspension of magnetic particles," United states Patent Office Filed Ser, vol. 315, 1965. 
[2] S. U. S. Choi, "Enhancing thermal conductivity of fluids with nanoparticles,"vol. 66, pp. 99-105, in Proceedings of the ASME International Mechanical Engineering Congress and Exposition, vol. 66, pp. 99-105, ASME Fluids Engineering Division, San Fransisco, CA, USA, 1995.

[3] C. SchererI and A. M. Figueiredo Neto, "Ferrofluids: properties and applications," Brazilian Journal of Physics, vol. 35, no. 3a, 2005.

[4] R. B. Bird, C. F. Curtiss, R. C. Armstrong, and O. Hassager, Dynamics of Polymeric Liquids, Wiley, New York, NY, USA, 1987.

[5] P. J. Carreau, "Rheological equations from molecular network theories," Transactions of the Society of Rheology, vol. 16, no. 1, pp. 99-127, 1972.

[6] J. L. Neuringer and R. E. Rosensweig, "Ferrohydrodynamics," Physics Fluids, vol. 7, no. 1, pp. 1927-1937, 1964.

[7] J. L. Neuringer, "Some viscous flows of a saturated ferro-fluid under the combined influence of thermal and magnetic field gradients," International Journal of Non-linear Mechanics, vol. 1, no. 2, pp. 123-137, 1966.

[8] H. I. Andersson and O. A. Valnes, "Flow of a heated ferrofluid over a stretching sheet in the presence of a magnetic dipole," Acta Mechanica, vol. 128, no. 1-2, pp. 39-47, 1998.

[9] G. H. R. Kefayati, "Natural convection of ferrofluid in a linearly heated cavity utilizing LBM," J. Mol. Liq.vol. 191, no. 1, pp. 1-9, 2014.

[10] T. Hayat, M. Ijaz Khan, M. Imtiaz, A. Alsaedi, and M. Waqas, "Similarity transformation approach for ferromagnetic mixed convection flow in the presence of chemically reactive magnetic dipole," Physics of Fluids, vol. 28, no. 10, Article ID 102003, 2016.

[11] A. Zeeshan, A. Majeed, and R. Ellahi, "Effect of magnetic dipole on viscous ferro-fluid past a stretching surface with thermal radiation," Journal of Molecular Liquids, vol. 215, no. 1, pp. 549-554, 2016.

[12] A. Majeed, A. Zeeshan, and R. Ellahi, "Unsteady ferromagnetic liquid flow and heat transfer analysis over a stretching sheet with the effect of dipole and prescribed heat flux," Journal of Molecular Liquids, vol. 223, pp. 528-533, 2016.

[13] A. Majeed, A. Zeeshan, and R. Ellahi, "Chemical reaction and heat transfer on boundary layer Maxwell Ferro-fluid flow under magnetic dipole with Soret and suction effects," Engineering Science and Technology, an International Journal, vol. 20, no. 1, pp. 1122-1128, 2017.

[14] M. Waqas, M. I. Khan, T. Hayat, and A. Alsaedi, "Numerical simulation for magneto Carreau nanofluid model with thermal radiation: a revised model," Computer Methods in Applied Mechanics and Engineering, vol. 324, pp. 640-653, 2017.

[15] K.-L. Hsiao, “To promote radiation electrical MHD activation energy thermal extrusion manufacturing system efficiency by using Carreau-Nanofluid with parameters control method," Energy, vol. 130, pp. 486-499, 2017.

[16] M. Khan, M. Azam, and A. Munir, "On unsteady FalknerSkan flow of MHD Carreau nanofluid past a static/moving wedge with convective surface condition," Journal of $\mathrm{Mo}$ lecular Liquids, vol. 230, pp. 48-58, 2017.

[17] M. Khan, M. Irfan, and W. A. Khan, "Numerical assessment of solar energy aspects on 3D magneto-Carreau nanofluid: a revised proposed relation," International Journal of Hydrogen Energy, vol. 42, no. 34, pp. 22054-22065, 2017.

[18] M. M. Bhatti, L. Phali, and C. M. Khalique, "Heat transfer effects on electro-magnetohydrodynamic Carreau fluid flow between two micro-parallel plates with
Darcy-Brinkman-Forchheimer medium," Archive of Applied Mechanics, pp. 1-13.

[19] D. Lu, M. Ramzan, N. U. Huda, J. D. Chung, and U. Farooq, "Nonlinear radiation effect on MHD Carreau nanofluid flow over a radially stretching surface with zero mass flux at the surface," Scientific Reports, vol. 8, no. 1, p. 3709, 2018.

[20] S. U. Khan and S. A. Shehzad, "Analysis for time-dependent flow of Carreau nanofluid over an accelerating surface with gyrotactic microorganisms: model for extrusion systems," Advances in Mechanical Engineering, vol. 11, no. 12, pp. 1-11, 2019.

[21] A. Hussain, Z. Muneer, M. Y. Malik, and S. Ali, "Formulating the behavior of thermal radiation and magnetic dipole effects on Darcy-Forchheimer grasped ferrofluid flow," Canadian Journal of Physics, vol. 97, no. 9, pp. 938-949, 2019.

[22] N. Kausar, S. Bilal, W. Tahir, and A. U. Nisa, "Physical prospective of induction of ferro-constituents in buoyancydriven magneto-nanofluid under the impact of magnetic dipole," Canadian Journal of Physics, vol. 97, no. 8, pp. 847-855, 2019.

[23] M. Waqas, S. Jabeen, T. Hayat, M. I. Khan, and A. Alsaedi, "Modeling and analysis for magnetic dipole impact in nonlinear thermally radiating Carreau nanofluid flow subject to heat generation," Journal of Magnetism and Magnetic Materials, vol. 485, no. 1, pp. 197-204, 2019.

[24] H. T. Basha, R. Sivaraj, A. Subramanyam Reddy, A. J. Chamkha, and H. M. Baskonus, "A numerical study of the ferromagnetic flow of Carreau nanofluid over a wedge, plate and stagnation point with a magnetic dipole," AIMS Mathematics, vol. 5, no. 5, pp. 4197-4219, 2020.

[25] M. Waqas, "A mathematical and computational framework for heat transfer analysis of ferromagnetic non-Newtonian liquid subjected to heterogeneous and homogeneous reactions," Journal of Magnetism and Magnetic Materials, vol. 493, no. 1, Article ID 165646, 2020.

[26] H. Waqas et al., "Significance of bioconvection in chemical reactive flow of magnetized Carreau.Yasuda nanofluid with thermal radiation and second.order slip," Journal of Thermal Analysis and Calorimetry, vol. 140, no. 1, pp. 1293-1306, 2020.

[27] K. Iqbal et al., "Magnetohydrodynamic thin film deposition of Carreau nanofluid over an unsteady stretching surface," Applied Physics A, vol. 126, p. 105, 2020.

[28] M. Taseer, S. Z. Alamri, H. Waqas, D. Habib, and R. Ellahi, "Bioconvection flow of magnetized Carreau nanofluid under the influence of slip over a wedge with motile microorganisms," Journal of Thermal Analysis and Calorimetry, vol. 143, pp. 945-957, 2020.

[29] A. Mishra, "MHD nonlinear radiative flow of Carreau nanofluid with variable chemical reaction: An approach to control global warming," Heat Transfer, vol. 50, pp. 1-22, 2020.

[30] M. Irfan, K. Rafiq, W. A. Khan, and M. Khan, "Numerical analysis of unsteady Carreau nanofluid flow with variable conductivity," Applied Nanoscience, vol. 10, no. 8, pp. 3075-3084, 2020.

[31] M. Irfan, M. Khan, W. A. Khan, and K. Rafiq, "Physical aspects of shear thinning/thickening behavior in radiative flow of magnetite Carreau nanofluid with nanoparticle mass flux conditions," Applied Nanoscience, vol. 10, no. 8, pp. 3021-3033, 2020.

[32] M. Irfan, Study of Brownian Motion and Thermophoretic Diffusion on Nonlinear Mixed Convection Flow of Carreau Nanofluid Subject to Variables Properties, Surfaces and Interfaces, Accepted, 2021. 
[33] M. M. Bhatti, L. Phali, and C. M. Khalique, "Heat transfer effects on electro-magnetohydrodynamic Carreau fluid flow between two micro-parallel plates with DarcyBrinkman-Forchheimer medium," Archive of Applied Mechanics, 2021.

[34] K. Jabeen, M. Mushtaq, and R. M. Akram, "Analysis of the MHD boundary layer flow over a nonlinear stretching sheet in a porous medium using semianalytical approaches," Mathematical Problems in Engineering, vol. 2020, no. 1, 9 pages, Article ID 3012854, 2020.

[35] K. Jabeen, M. Mushtaq, and R. M. Akram Muntazir, "Analysis of MHD fluids around a linearly stretching sheet in porous media with thermophoresis, radiation, and chemical reaction," Mathematical Problems in Engineering, vol. 2020, no. 1, 14 pages, Article ID 9685482, 2020.

[36] M. B. Arain, M. M. Bhatti, A. Zeeshan, T. Saeed, and A. Hobiny, "Analysis of arrhenius kinetics on multiphase flow between a pair of rotating circular plates," Mathematical Problems in Engineering, vol. 2020, Article ID 2749105, 17 pages, 2020. 